\title{
INDICADORES EPIDEMIOLÓGICOS APLICÁVEIS A ESTUDOS SOBRE A ASSOCIAÇÃO ENTRE SANEAMENTO E SAÚDE DE BASE MUNICIPAL
}

\author{
EPIDEMIOLOGICAL INDICATORS APPLIED TO STUDIES ABOUT ASSOCIATION \\ BETWEEN WATER SUPPLY AND SANITATION AND HEALTH BASED ON \\ DATA AT MUNICIPAL LEVEL
}

\section{SILVANO SILVÉRIO DA COSTA}

Engenheiro Civil (FE- FUMEC). Mestre em Tecnologia Ambiental e Recursos Hídricos (UnB). Diretor de Operação e Manutenção do SAAE de Guarulhos-SP. Presidente da ASSEMAE

\section{LÉO HELLER}

Engenheiro Civil (UFMG). Mestre em Engenharia Sanitária (UFMG). Doutor em Epidemiologia (UFMG). Professor do Departamento de Engenharia Sanitária e Ambiental - UFMG

\section{CRISTina Celia Silveira Brandão}

Engenheira Química (UFBA). Mestre em Engenharia Química (COPPE/UFRJ). Doutora em Engenharia Ambiental (Imperial College - Universidade de Londres). Professora do Departamento de Engenharia Civil e Ambiental da Universidade de Brasília - UnB

\section{ENRICO ANTÔNIO COLOSIMO}

Engenheiro Eletricista (UFMG). Mestre em Estatística (UNICAMP). Doutor em Estatística (University of Wisconsin, Estados Unidos). Professor do Departamento de Estatística - UFMG

Recebido: 05/08/04 Aceito: 14/02/05

\section{RESUMO}

É relativamente recente no país a prática da vigilância ambiental em saúde, sendo que a vigilância da qualidade da água para consumo humano, parte integrante da vigilância ambiental em saúde, ressente-se de estudos e pesquisas que forneçam suporte científico à formulação de instrumentos para a prática de tal vigilância. Nesse campo, a formulação e seleção de indicadores epidemiológicos constituem atividade essencial para representar os efeitos da insuficiência das ações de saneamento sobre a saúde humana e, portanto, como ferramenta para a vigilância e orientação de programas e planos de alocação de recursos em saneamento. O presente artigo é parte de um trabalho mais abrangente, elaborado para selecionar indicadores sanitários como sentinelas na prevenção e controle de doenças e agravos relacionados ao saneamento. Neste artigo são enfocados os indicadores epidemiológicos utilizados naquele estudo, apresentando a revisão bibliográfica, resultados da estatística descritiva desses indicadores, a discussão e conclusōes sobre os indicadores epidemiológicos mais apropriados para serem utilizados emestudos dessa natureza. Destacam-se, como indicadores convenientes, a mortalidade infantil, a mortalidade até cinco anos por doenças diarréicas e morbidade até cinco anos por doenças diarréicas com base na MDDA monitorização de doenças diarréicas agudas.

PALAVRAS-CHAVE: Indicadores epidemiológicos, saneamento, saúde ambiental, diarréia, mortalidade infantil.

\section{ABSTRACT}

Environmental health surveillance has been recently implemented in Brazil. Drinking water quality surveillance, a fundamental aspect of the environmental health surveillance, lacks specific studies and research to provide scientific background for its practice. In this field, the identification and selection of epidemiological indicators are essential to provide means to evaluate the impact of deficiencies of water supply and sanitation on human health. So, these indicators are powerful tools for the establishment of drinking water surveillance programs, as well as for identification of priorities, rational planning and funding of water supply and sanitation projects. The present paper is part of a broader study on the selection of sanitary indicators to be used as "sentinel indicators" for prevention and control of diseases associated to water and sanitation. In this context, this paper presents a briefliterature review, descriptive statistics of some epidemiological indicators collected from municipalities of 4 Brazilian States, as well as a discussion on the applicability of these indicators in studies of this nature. Among the epidemiological indicators evaluated, infant mortality, diarrhoea mortality in children under 5 years old and the diarrhoea morbidity in children under 5 years old based on data from the Brazilian Monitoring of Acute Diarrhoeal Disease program seem to be the more adequate.

KEYWORDS: Epidemiological indicators, water and sanitation, environmental health, diarrhoea, infant mortality. 


\section{INTRODUÇÃO}

Prüss et al (2002) estimam que a diarréia é responsável por $4,3 \%$ dos anos de vida perdidos ou com incapacitação (DALY) no mundo e que $88 \%$ desta carga de doenças é atribuída ao abastecimento de água, esgotamento sanitário e higiene inadequados. A maior concentração dessa carga localiza-se em crianças dos países em desenvolvimento, cuja situação do saneamento encontra-se extremamente vulnerável, não se vislumbrando perspectivas sustentadas e continuadas de reversão do quadro.

Um dos desafios presentes consiste na definição de indicadores epidemiológicos e sanitários que permitam nortear as açōes e empreender avaliaçōes no campo do saneamento. Especialmente nos países em desenvolvimento, as áreas de saneamento e de saúde, ainda que disponham, respectivamente, de um conjunto de indicadores sanitários e epidemiológicos, não os utilizam de forma sistemática e integrada, para fornecer suporte qualificado às suas açôes, na meta de universalizar com eqüidade $o$ atendimento. Tais indicadores, além de seu potencial em representar os efeitos da insuficiência das ações de saneamento sobre a saúde humana, podem constituir ferramenta para a vigilância e para a orientação de programas e planos de alocação de recursos em saneamento.

Esse desafio, portanto, inclui uma mais profícua integração dos setores de saneamento e meio ambiente com o setor saúde, sendo que as informações a respeito da realidade desses setores são a base fundamental para tal integração. As vigilâncias epidemiológica, ambiental e sanitária, utilizando essas informações, agindo e demandando medidas de correção aos serviços de saneamento, poderiam contribuir significativamente para a redução das ocorrências de doenças e agravos decorrentes da veiculação hídrica.

O setor saúde, no Brasil, conta atualmente com programas institucionais que estão sendo desenvolvidos no sentido de integrar as vigilâncias sanitária, epidemiológica e ambiental, na busca da Vigilância em Saúde. Nesta perspectiva, a Vigilância da Qualidade da Água para Consumo Humano, parte integrante da Vigilância Ambiental em Saúde, tem como objetivo integrar o setor saúde com os setores de saneamento e meio ambiente. Tal integração deve ocorrer a partir das ações e informaçôes de vigilância ambiental e epidemiológica -, com as ações e informações relativas à prestação dos serviços, nos aspectos da cobertura e da qualidade do atendimento.

Indicadores epidemiológicos são importantes para representar os efeitos das ações de saneamento - ou da sua insuficiência - na saúde humana e constituem, portanto, ferramentas fundamentais para a vigilância ambiental em saúde e para orientar programas e planos de alocação de recursos em saneamento ambiental no país.

Os projetos de abastecimento de água e esgotamento sanitário podem influenciar um número amplo de variáveis relativas a doenças ou ao estado de saúde (Briscoe et al, 1986), dentre as quais destacam-se a morbi-mortalidade devido à diarréia, o estado nutricional, nematóides intestinais, infecção dos olhos e infecção da pele.

A maioria dos estudos que avaliam o impacto da melhoria do abastecimento de água e esgotamento sanitário na saúde de crianças centra-se em um ou mais dos seguintes grupos de indicadores: ocorrência de diarréia, presença de parasitas e patogênicos nas fezes, estado nutricional e mortalidade na infância (Heller, 1997). Todos esses indicadores foram identificados em uma revisão de 67 estudos em 28 países (Esrey et al, 1985).

A escolha de uma variável ou de um indicador, que reflita o estado de saúde de um grupo populacional, deve conciliar o compromisso entre a necessidade de efetivamente expressar a condição da saúde coletiva, por um lado, e a sua adequação à pesquisa em questão, por outro. Segundo Briscoe et al (1986), essa escolha será influenciada pela sua importância para a saúde pública; pela sua validade e confiabilidade nos instrumentos para medir a variável e pela sua capacidade de resposta às alterações das condiçōes de abastecimento de água e esgotamento sanitário.

Nessa discussão, o emprego do indicador morbidade por doenças diarréicas tem sido referendado por trabalhos que estabelecem roteiros metodológicos para os estudos de impacto de saneamento sobre a saúde (Heller, 1997). Embora reúna com vantagens aqueles critérios sugeridos, é preciso reconhecer limitaçôes no indicador. Essas são advindas de dificuldades na obtenção da informação de forma padronizada - com confiabilidade -, em vista tanto das variações da compreensão popular sobre a definição de diarréia quanto à possibilidade de viés na informação, quando resulta de inquérito populacional, com indagação sobre sua ocorrência às mães ou responsáveis por crianças.

Indicadores de mortalidade, a exemplo da mortalidade por diarréia, também mostram-se como alternativa para adoção nas situações em análise, a despeito de algumas limitaçóes apontadas por Briscoe et al (1986), que o consideram "uma variável de indiscutível importância para a saúde pública, porém com limitaçôes na confiabilidade e na validade dos dados obtidos, quer nas estatísticas oficiais, quer em inquéritos domiciliares".

Algumas populações são particularmente sensíveis às diversas patologias. As crianças de até um ano de idade são susceptíveis a diversas doenças, inclusive aquelas causadas por fatores ambientais. Idosos sofrem não só as conseqüências de toda uma exposição a uma série de fatores químicos, exposiçōes profissionais, etc., como são mais suscetíveis, pela diminuição da resistência orgânica, para uma série de doenças (respiratórias, fraturas, acidentes e outras) (Câmara et al, 2000).

Particularmente em relação às crianças, Câmara et al (2000) argumentam que crianças menores de um ano, com algumas exceções, devem sobreviver sem maiores problemas, a não ser quando acidentes ambientais ou hospitalares venham a se interpor no curso natural do seu desenvolvimento. Nesse caso, a mortalidade infantil pode chegar a níveis muito elevados, refletindo um ambiente hostil (desnutrição, vacinação incompleta ou ausente, saneamento parcial ou inexistente, atendimento médico hospitalar ineficaz durante a gestação, parto ou ambos, etc).

Segundo Esrey et al (1990), a maioria dos estudos desenvolvidos em diferentes países avalia os impactos do abastecimento de água e esgotamento sanitário na saúde de crianças. Ratificando a conclusão de Esrey et al (1990), nas referências bibliográficas consultadas sobre a avaliação do impacto do abastecimento de água e do esgotamento sanitário sobre a saúde, a maioria emprega a morbi-mortalidade de enfermidade em crianças até os cinco anos de idade.

Nesse contexto, o presente artigo enfoca os indicadores epidemiológicos utilizados em um trabalho mais abrangente desenvolvido por Costa (2002), visando selecionar indicadores sanitários como potenciais "indicadores sentinelas" na prevenção e controle de doenças e agravos relacionados ao saneamento. Assim, são aqui descritos e discu- 
tidos os indicadores epidemiológicos mais apropriados para serem utilizados em estudos dessa natureza.

\section{METODOLOGIA}

\section{Estados e municípios analisados}

Para o estudo original (Costa, 2002) foram escolhidos quatro estados brasileiros - Pernambuco, Bahia, Paraná e Rio Grande do Sul. A escolha foi condicionada pelo estágio mais avançado em que, à época, encontrava-se a vigilância da qualidade da água para consumo humano nas Secretarias de Saúde daqueles estados, quando comparado com o dos demais estados da federação. Nesses estados, foi implantado experimentalmente o SISAGUA - Sistema de Informação em Vigilância da Qualidade da Água para Consumo Humano, pelo então CGVAM/FUNASA do Ministério da Saúde, hoje CGVAM/SVS do mesmo Ministério da Saúde.

\section{Indicadores epidemiológicos adotados}

Com base na discussão apresentada, para avaliar a influência da melhoria do abastecimento de água e do esgotamento sanitário na saúde, foram selecionados indicadores de morbidade por doenças diarréicas (cólera, diarréia, gastroenterite de origem infecciosa presumível, e outras doenças diarréicas de origem infecciosa presumível), de mortalidade geral e de mortalidade por doenças diarréicas.

Quanto à faixa etária, os indicadores epidemiológicos foram analisados para crianças menores de um ano e menores de cinco anos, em função das diversas citações mostrando que as açōes de melhoria das condiçôes de saneamento refletem-se mais especificamente na saúde das crianças.

Os indicadores relativos à mortalidade foram calculados para mil nascidos vivos, enquanto que os de morbidade foram adotados para 100.000 nascidos vivos.

Inicialmente os indicadores epidemiológicos utilizados para a análise foram:

- Mortalidade

o mortalidade por doenças diarréicas para crianças menores de cinco anos por mil nascidos vivos (MORTDD5N); o mortalidade por todas as causas para crianças menores de cinco anos por mil nascidos vivos (MORTTC5N); o mortalidade infantil - menores de um ano e por mil nascidos vivos (MINF);

- Morbidade

- morbidade por doenças diarréicas para crianças menores de cinco anos e por 100.000 nascidos vivos, com base em dados extraídos do Sistema de Internaçōes Hospitalares SIH/SUS (MORBDD5S).

o morbidade por doenças diarréicas para crianças menores de cinco anos e por 100.000 nascidos vivos, com base em dados extraídos da Monitorização das Doenças Diarréicas Agudas MDDA/SES (MORBDD5M).

Após a elaboração do histograma de distribuição de frequiências dos indicadores, apresentados no item RESULTADOS e DISCUSSÃO, foi possível verificar que a freqüência dos indicadores de mortalidade por doenças diarréicas (MORTDD5N) e de morbidade por doenças diarréicas segundo dados do SIH/SUS (MORBDD5S) não apresentavam distribuição normal, em função da quantidade de valores nulos, optando-se por tratá-los como variáveis dicotômicas, procedendo-se assim à conversão dessas variáveis. Após a transformação, as variáveis passaram a ser denominadas MTDD5DIC (mortalidade por doenças diarréicas) e MBDD5DIC (morbidade por doenças diarréicas extraídas do SIH/SUS). Os procedimentos de transformação dessas variáveis são detalhados adiante.

Os indicadores epidemiológicos podem ser, assim, sintetizados:

\section{- Mortalidade}

o mortalidade por doenças diarréicas para crianças menores de cinco anos - variável dicotômica (MTDD5DIC);

o mortalidade por todas as causas para crianças menores de cinco anos por mil nascidos vivos (MORTTC5N);

o mortalidade infantil - menores de um ano e por mil nascidos vivos (MINF);

\section{- Morbidade}

o morbidade por doenças diarréicas para crianças menores de cinco anos, com base em dados extraídos do Sistema de Internaçôes Hospitalares SIH/SUS variável dicotômica (MBDD5DIC);

o morbidade por doenças diarréicas para crianças menores de cinco anos e por 100.000 nascidos vivos, com base em dados extraídos da Monitorização das Doenças Diarréicas Agudas-MDDA/SES (MORBDD5M).

\section{Fórmulas de cálculo}

A Tabela 1 apresenta as principais fórmulas para o cálculo dos indicadores epidemiológicos.

\section{Breve descrição da análise estatística realizada no estudo original}

\section{Concepção geral}

Inicialmente, foi construído banco de dados composto por todos os indicadores epidemiológicos, sanitários e sociais por município. A partir do banco de dados desenvolveram-se análises estatísticas dos indicadores para todos os municípios.

Análise descritiva dos indicadores

Inicialmente foi realizada análise da distribuição de freqüência de todos os indicadores, com vistas, basicamente, a subsidiar as análises de regressão.

Passou-se então à análise descritiva, a partir dos seguintes parâmetros:

- número de dados;

- média;

- variância;

- desvio padrão;

- variação (mínimo e máximo).

Adicionalmente, foram construídos gráficos de dispersão, associando as variáveis uma a uma.

\section{Análise de regressão simples}

A análise de regressão simples foi utilizada para analisar, de forma preliminar, o comportamento das variáveis de mesma natureza (indicadores epidemiológicos, sanitários e sociais entre si) e de natureza diferente (indicadores epidemiológicos versus sanitários e sociais).

As variáveis epidemiológicas foram classificadas como variáveis dependentes, enquanto as variáveis sanitárias e sociais foram tratadas como independentes.

Para a análise de regressão simples foram utilizados dois tipos de análise de regressão: a regressão linear e a logística. A primeira foi empregada para aquelas variáveis dependentes caracterizadas como variáveis contínuas (MORTTC5N, MINF e MORBDD5M), a quais apresentaram distribuição normal. A segunda foi adotada para as variáveis dicotômicas MTDD5DIC e MBDD5DIC. 
Tabela I - Cálculo dos indicadores epidemiológicos

\begin{tabular}{|c|c|}
\hline Indicador & Fórmula de cálculo (por município) \\
\hline $\begin{array}{l}\text { MORTDD } 5 \mathrm{~N} \text { - mortalidade por doenças diarréicas (cólera, } \\
\text { diarréia, gastroenterite de origem infecciosa presumível e } \\
\text { outras doenças diarréicas de origem infecciosa presumível) } \\
\text { em crianças menores de cinco anos por mil }\end{array}$ & $\begin{array}{l}\text { \{[óbitos por doenças diarréicas até cinco anos } \\
\left.\left.(\text { SIM-1999)/ nascidos vivos (SINASC-1999) }]^{*} 1000\right)\right\}\end{array}$ \\
\hline $\begin{array}{l}\text { MTDD5DIC - mortalidade por doenças diarréicas (cólera, } \\
\text { diarréia, gastroenterite de origem infecciosa presumível e } \\
\text { outras doenças diarréicas de origem infecciosa presumível) } \\
\text { em crianças menores de cinco anos- variável dicotômica }\end{array}$ & $\begin{array}{l}\text { MTDD5DIC }=0, \text { se } \text { MORTDD } 5 \mathrm{~N}=0 \\
\text { MTDD5DIC }=1, \text { se } \text { MORTDD5N }>0\end{array}$ \\
\hline $\begin{array}{l}\text { MORTTC } 5 \mathrm{~N} \text { - mortalidade por todas as causas em } \\
\text { crianças menores de cinco anos por mil }\end{array}$ & $\begin{array}{l}\{[\text { óbitos por todas as causas até cinco anos } \\
\left.\left.(\text { SIM-1999)/ nascidos vivos (SINASC-1999) }]^{*} 1000\right)\right\}\end{array}$ \\
\hline $\begin{array}{l}\text { MINF - Mortalidade infantil em crianças menores de um } \\
\text { ano por mil }\end{array}$ & $\begin{array}{l}\text { Indicador obtido diretamente do site do DATASUS/MS, } \\
\text { sendo que metodologia de cálculo foi proposta } \\
\text { por Simóes (1999) }\end{array}$ \\
\hline $\begin{array}{l}\text { MORBDD } 5 S \text { - morbidade por doenças diarréicas (cólera, } \\
\text { diarréia, gastroenterite de origem infecciosa presumível e } \\
\text { outras doenças diarréicas de origem infecciosa presumível) } \\
\text { em crianças menores de cinco anos por } 100 \text { mil (dados } \\
\text { SIH/SUS) }\end{array}$ & $\begin{array}{l}\{[\Sigma \text { do número de internaçōes por doenças diarréicas dos } \\
\text { meses de abril/2000 a março/2001 por município } \\
\text { (SIH/DATASUS)/ nascidos vivos } \\
\left.\text { (SINASC-1999) }]^{*} 100.000\right\}\end{array}$ \\
\hline $\begin{array}{l}\text { MBDD5DIC - morbidade por doenças diarréicas (cólera, } \\
\text { diarréia, gastroenterite de origem infecciosa presumível e } \\
\text { outras doenças diarréicas de origem infecciosa presumível) } \\
\text { em crianças menores de cinco anos - variável dicotômica } \\
\text { (dados SIH/SUS) }\end{array}$ & $\begin{array}{l}\text { MBDD5DIC }=0, \text { se } M O R B D D 5 S=0 \\
\text { MBDD5DIC }=1, \text { se } \text { MORBDD5S }>0\end{array}$ \\
\hline $\begin{array}{l}\text { MORBDD } 5 \mathrm{M} \text { - morbidade por doenças diarréicas (cólera, } \\
\text { diarréia, gastroenterite de origem infecciosa presumível e } \\
\text { outras doenças diarréicas de origem infecciosa presumível) } \\
\text { em crianças menores de cinco anos por } 100 \text { mil (dados } \\
\text { MDDA) }\end{array}$ & $\begin{array}{l}\{[\Sigma \text { do número de casos registrados por unidade de saúde } \\
\text { por município por doenças diarréicas dos meses do ano } \\
\left.2000(\mathrm{SES}) / \text { nascidos vivos (SINASC-1999) }]^{*} 100.000\right\}\end{array}$ \\
\hline
\end{tabular}

\section{Análise de regressão multivariada}

Nas análises multivariadas também foram utilizadas as regressōes linear elogística, para as mesmas variáveis. Após a seleção das variáveis com significância na regressão simples (variáveis com p valor $\leq 0,20$ ), foram desenvolvidas as regressōes multivariadas para avaliar a associação entre as variáveis epidemiológicas e sanitárias, considerando a influência das variáveis sociais intervenientes.

\section{RESULTADOS}

À exceção da morbidade por doenças diarréicas, com notificação de casos pelas unidades de saúde nos municípios com monitorização de doenças diarréicas agudas-MDDA, onde se conseguiram obter indicadores apenas no estado de Pernambuco e poucos municípios dos estados da Bahia e do Rio Grande do Sul, foi possível reunir dados para a constru- ção dos indicadores epidemiológicos para a maioria dos municípios.

A Tabela 2 apresenta, por estado, o número de municípios com indicadores epidemiológicos identificados nos bancos de dados.

\section{Análise descritiva dos indicadores}

São apresentados, nas Figuras 1 a 5 , os histogramas de distribuição de freqüência dos indicadores epidemiológicos.

\section{Regressão simples}

Na primeira análise, foram associados os indicadores de mesma natureza entre si, e na segunda foram feitas análises de regressão dos indicadores epidemiológicos versus os sanitários e sociais. A Tabela 3 apresenta matriz com os coeficientes de Pearson ( $r$ ) resultantes das correlaçóes entre indicadores epidemiológicos.

\section{DISCUSSÃO}

\section{O problema da sub- notificação}

Destaca-se inicialmente uma fragilidade presente na maioria dos indicadores de morbi-mortalidade: a sub-notificação, tanto do número de internaçôes ou de óbitos, quanto do número oficial de nascimentos.

Sobre essa questão, Simões (1999) aponta que a existência de omissões decorrentes do sub-registro de óbitos, principalmente daqueles menores de um ano, tem inviabilizado, em muitos casos, a utilização deste instrumento de coleta no cálculo direto da mortalidade, necessitando-se recorrer a procedimentos técnicos para corrigir essas distorções. O problema é mais grave naqueles estados e regiōes social e economicamente menos desenvolvidos, onde a população rural representa uma proporção importante da po- 
Tabela 2 - Número de municípios com indicadores epidemiológicos identificados por estado

\begin{tabular}{cccccccc}
\hline Estado & $\begin{array}{c}\text { Número } \\
\text { municípios } \\
\text { do estado }\end{array}$ & $\begin{array}{c}\text { Número } \\
\text { municípios } \\
\text { SISAGUA }\end{array}$ & $\begin{array}{c}\text { Mortalidade } \\
\text { doenças diarréicas } \\
\text { até cinco anos }\end{array}$ & $\begin{array}{c}\text { Mortalidade } \\
\text { todas as causas até } \\
\text { cinco anos }\end{array}$ & $\begin{array}{c}\text { Morbidade doenças } \\
\text { diarréicas - } \\
\text { Internaçóes }\end{array}$ & $\begin{array}{c}\text { Mortalidade } \\
\text { infantil }\end{array}$ & $\begin{array}{c}\text { Morbidade } \\
\text { doenças diarréicas - } \\
\text { MDDA }\end{array}$ \\
\hline BA & 424 & 116 & 116 & 116 & 116 & 116 & 22 \\
PR & 409 & 194 & 194 & 193 & 193 & 194 & 0 \\
PE & 193 & 86 & 86 & 85 & 86 & 86 & 85 \\
RS & 475 & 104 & 104 & 104 & 104 & 104 & 5 \\
\hline
\end{tabular}

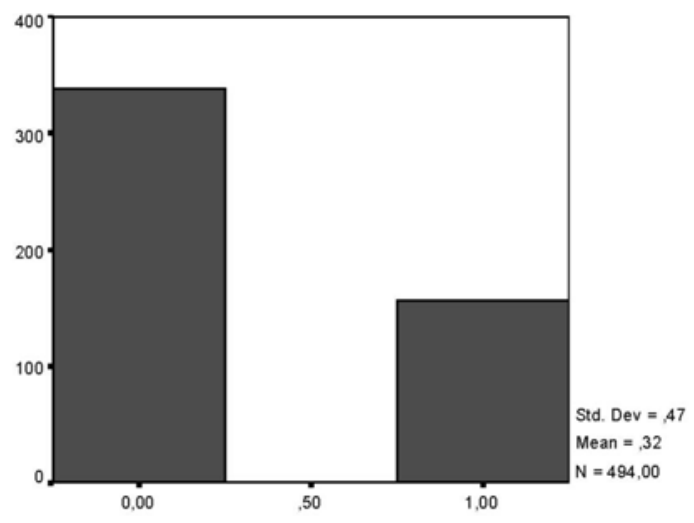

MTDD5DIC

Figura I - Histograma de distribuição de freqüência: mortalidade por doenças diarréicas em crianças menores de 5 anos - variável dicotômica

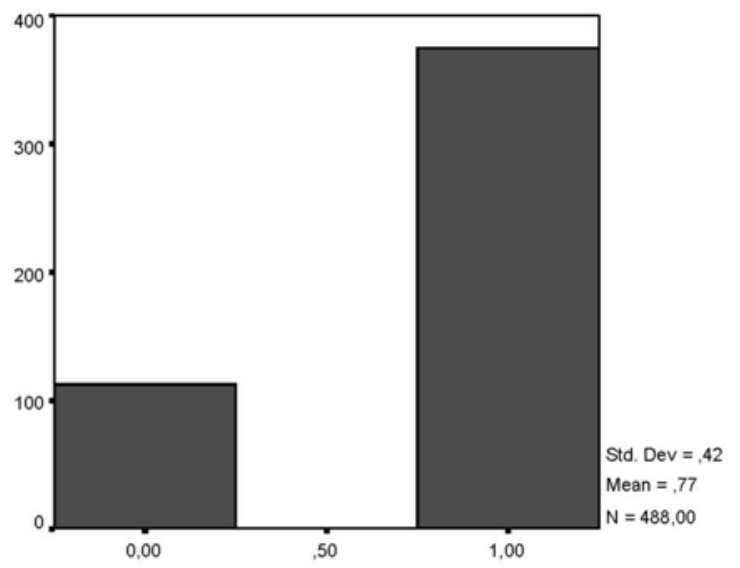

MBDD5DIC

Figura 3 - Histograma de distribuição de freqüência: morbidade por doenças diarréicas em crianças menores de 5 anos - internações hospitalares variável dicotômica

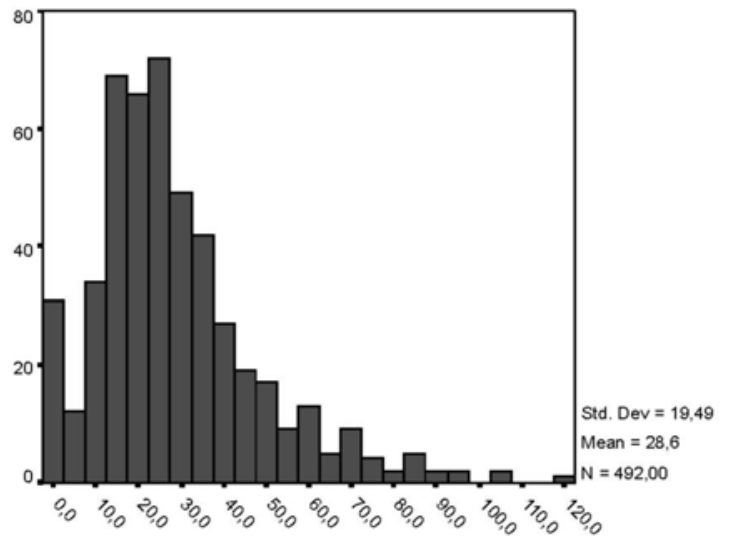

MORTTC5N

Figura 2 - Histograma de distribuição de freqüência: mortalidade por todas as causas em crianças menores de 5 anos

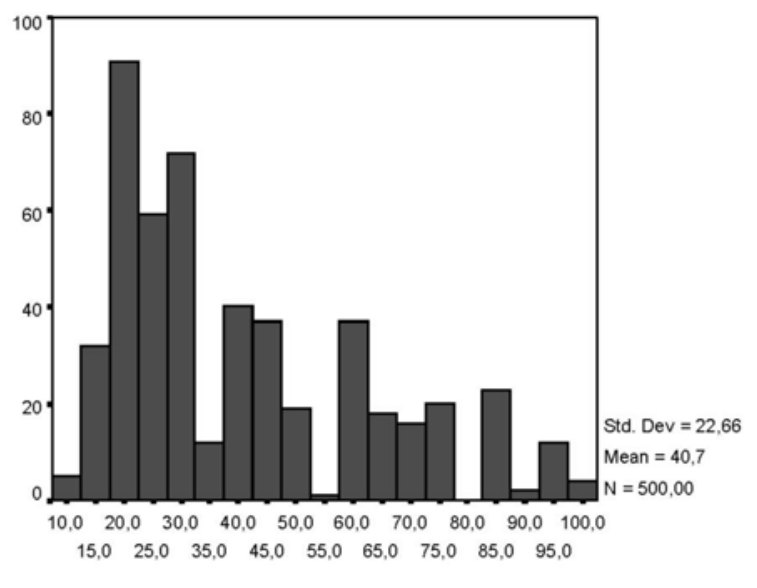

MINF

Figura 4 - Histograma distribuição de freqüência: mortalidade infantil 


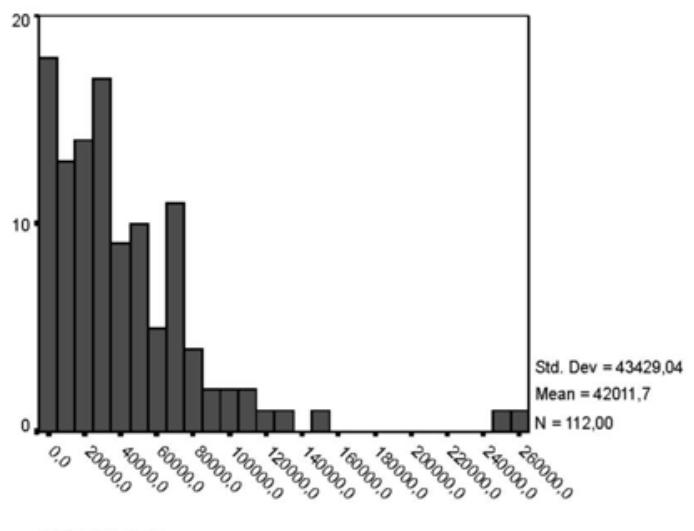

MORBDD5M

\section{Figura 5 - Histograma de freqüência: morbidade por doenças diarréicas em crianças menores de 5 anos - notificação no MDDA}

Tabela 3 - Matriz de correlação entre indicadores epidemiológicos - coeficiente de Pearson ( $r$ )

\begin{tabular}{lccccc}
\hline & $\begin{array}{c}\text { Mortalidade } \\
\text { todas as } \\
\text { causas }\end{array}$ & $\begin{array}{c}\text { Mortalidade } \\
\text { infantil }\end{array}$ & $\begin{array}{c}\text { Morbidade } \\
\text { doenças } \\
\text { diarréicas - } \\
\text { MDDA }\end{array}$ & $\begin{array}{c}\text { Mortalidade } \\
\text { doenças } \\
\text { diarréicas }\end{array}$ & $\begin{array}{c}\text { Morbidade } \\
\text { doenças } \\
\text { diarréicas - } \\
\text { Internaçóes }\end{array}$ \\
\hline $\begin{array}{l}\text { Mortalidade todas as causas } \\
\text { Mortalidade infantil }\end{array}$ & 1 & 0,52 & 0,09 & 0,39 & 0,04 \\
$\begin{array}{l}\text { Morbidade doenças } \\
\text { diarréicas - MDDA }\end{array}$ & 1 & 0,04 & 0,51 & 0,06 \\
$\begin{array}{l}\text { Mortalidade doenças } \\
\text { diarréicas }\end{array}$ & & 1 & 0,23 & 0,33 \\
$\begin{array}{l}\text { Morbidade doenças } \\
\text { diarréicas - Internaçóes }\end{array}$ & & & 1 & 0,09 \\
\hline
\end{tabular}

pulação, como é o caso das regiōes Norte, Nordeste e Centro Oeste. Na região centrosul do país os problemas de cobertura das notificações não são tão significativos.

\section{- Sub-registros de nascimento}

Apresentam-se na Tabela 4 os sub-registros relativos ao nascimento nos estados objeto do presente estudo.

A melhor qualidade de registros de nascimento ocorre nos estados da Região Sudeste e nos estados da Região Sul - o Rio Grande do Sul, por exemplo, exibe valores de subnotificação de registro civil de nascimento da ordem de $10 \%$.

Existe uma tendência em se adotarem dados do Sistema Nacional de Nascidos Vivos - SINASC, em detrimento dos dados estatísticos de registro civil. No país como um todo, $18 \%$ dos nascimentos deixam de ser computados pelo SINASC, contra 31\% pelo Registro Civil.
Nos estados do RJ, SP, PR, RS e GO, onde o SINASC está implantado em todos os municípios, é possível afirmar que os valores observados no número de nascimentos coletados pelo Sistema sejam mais corretos. Destaca-se que para os indicadores epidemiológicos, à exceção da mortalidade infantil, foram utilizados dados de nascidos vivos do SINASC nos seus denominadores.

\section{- Sub-registro de óbitos}

Na Tabela 5 são mostrados os subregistros referentes a óbitos infantis.

Verifica-se que o problema de sub-registros de óbitos é maior que o relativo ao nascimento, sendo que na média do país é de 19,11\%.

\section{Indicadores epidemiológicos}

\section{- Mortalidade infantil}

Os indicadores de mortalidade infantil para cada município foram obtidos do sítio do Ministério da Saúde, cujo cálculo adotou a metodologia proposta por Simões (1999), baseada em técnicas demográficas utilizando informações sobre sobrevivência de filhos nascidos vivos nos Censos Demográficos e nas PNAD - Pesquisas Nacionais de Amostragem Domiciliar.

A mortalidade infantil, apesar de não considerar uma parte significativa de crianças passíveis de serem afetadas pelas questôes do saneamento - as maiores de um ano -, mostra-se como um importante indicador epidemiológico para analisar o impacto das intervençôes do saneamento na saúde humana. Trata-se de um indicador universal, padronizado há muitos anos e utilizado em todo o mundo para aferir o nível de desenvolvimento da sociedade.

Ainda que os indicadores refiram-se ao ano de 1998 e os indicadores sanitários sejam relativos ao ano 2000 , é possível constatar que, das variaçõos observadas na evolução da mortalidade infantil no período 1989-1998, não é freqüente se observarem elevadas variaçôes diferenciais entre municípios, para pequenos períodos de defasagem.

A Tabela 6 mostra os valores médios de mortalidade infantil dos estados e a média do Brasil e das regióes, apresentadas para compreensão da situação do indicador regionalmente.

Quanto à abrangência, registra-se que a mortalidade infantil encontra-se disponível para todos os municípios que fizeram parte do estudo.

Os indicadores sanitários que apresentaram associação estatística significativa e em sentido inverso à mortalidade infantil foram a turbidez (variável dicotômica que diferencia os municípios que têm $100 \%$ das amostras com turbidez dentro dos padrōes na rede de distribuição de água daqueles que têm percentual diferente de $100 \%$ ) e o percentual da população do município com coleta regular de lixo. Surpreendentemente, a cobertura por coleta de esgotos e por sistema de abastecimento de água apresentou relação direta com o valor do indicador, o que, em caráter especulativo, foi atribuído a fatores como a maior geração e exposição de esgotos no meio urba- 
Tabela 4 - Comparação entre o sub-registro de nascimento entre o Civil e SINASC

\begin{tabular}{ccc}
\hline Estado & \multicolumn{2}{c}{ Sub-Registro } \\
& Registro & SINASC \\
& Civil-1995 & 1996 \\
\hline Bahia & 36,60 & 31,44 \\
Pernambuco & 47,64 & 6,29 \\
Paraná & 20,56 & $-1,83$ \\
Rio Grande do Sul & 10,22 & $-3,63$ \\
\hline
\end{tabular}

Fonte: Simões (1999)
Tabela 5 - Sub-registro de óbitos infantis (menores de um ano)

\begin{tabular}{lcc}
\hline \multicolumn{1}{c}{ Estado } & \multicolumn{2}{c}{ Sub-Registro Óbitos(\%) } \\
& $\begin{array}{c}\text { Registro } \\
\text { Civil - 1995 }\end{array}$ & CENEPI/MS - \\
& SIM/1996 \\
\hline Bahia & 39,25 & 66,01 \\
Pernambuco & 25,21 & 43,32 \\
Paraná & 24,26 \\
Rio Grande do Sul & 2,41 & 5,13 \\
\hline Fonte: Simões (1999) & &
\end{tabular}

Tabela 6- Média da mortalidade infantil, no ano de 1998, para os estados de interesse, para o Brasil e para as regiões (por mil nascidos vivos)

Valores médios de mortalidade infantil

Bahia Pernambuco Paraná Rio Grande do Sul Brasil

\begin{tabular}{lccccc}
\hline $\begin{array}{l}\text { Municípios } \\
\text { considerados }\end{array}$ & 54 & 77 & 29 & 19 & \\
Estado & 51 & 62 & 28 & 19 & 36,10 \\
Região & 58 & 58 & 22 & 22 & \\
\hline
\end{tabular}

no, aumentando riscos de transmissão de enfermidades na infância.

\section{- Mortalidade até cinco anos por todas as causas}

Esse indicador é também vulnerável à sub-notificação, tanto em relação aos óbitos quanto em relação aos registros de nascidos vivos, maior nos estados do Nordeste quando comparados com os da região Sul.

A mortalidade por todas as causas em crianças menores de cinco anos é um indicador complementar à mortalidade infantil, na medida em que considera também o número de óbitos em crianças entre um e quatro anos. De acordo com a revisão da literatura, essa faixa etária é muito afetada pelas inadequaçôes ou falta de serviços de saneamento. Por outro lado, no indicador mortalidade por todas as causas, vários outros fatores de risco, não só decorrentes das condições sanitárias adversas, podem estar presentes.

Chama a atenção o número de municípios que não apresentaram registros de óbitos nessa faixa etária: dos $500 \mathrm{mu}-$ nicípios estudados, aproximadamente 30 (6\%). Esse número pode estar mais ou menos influenciado pela sub-notificação referida anteriormente. Essa constatação fica mais evidenciada quando se analisa a Tabela 7. A diferença entre a mortalidade infantil e a mortalidade até cinco anos, em que pese a diferença da metodologia de cálculo e das faixas etárias, é muito menor nos estados do Paraná e Rio Grande do Sul, que nos estados da Bahia e Pernambuco.

A despeito da quantidade de municípios que não apresentaram registros de óbitos por mil nascidos vivos, a correlação entre a mortalidade por todas as causas em crianças menores de cinco anos com a mortalidade infantil poderia ser classificada como moderada $(r=0,52)$. Por um lado, essa correlação pode ser influenciada pela qualidade das notificaçōes de óbitos e de registros dos municípios da região Sul, cuja sub-notificação é bem menor que nos estados do Nordeste, e, por outro, pelo fato de terem sido considerados na mortalidade até cinco anos os óbitos de crianças entre um e quatro anos, o que faz aumentar a mortalidade e compensar a sub-notificação já referida.

O indicador sanitário que apresentou associação estatística significativa e em sentido inverso à mortalidade por todas as causas até cinco anos foi o percentual da população do município com coleta regular de lixo. Por outro lado, cobertura por coleta de esgotos e cobertura por banheiros mostraram-se estatisticamente associadas, porém com relação direta, com o valor do indicador, o que, em caráter especulativo, da mesma forma como na análise da mortalidade infantil, foi atribuído a fatores como a maior geração e exposição de esgotos no meio urbano, aumentando riscos de transmissão de enfermidades na infância.

\section{- Mortalidade até cinco anos por doenças diarréicas}

É notável, nesse indicador, a proporção significativa de municípios que não apresentaram óbitos por doenças diarréicas. Dos 500 municípios, apenas $150(30 \%)$ apresentaram óbitos por esta causa. Possíveis explicaçōes são:

· sub-notificaçóes, conforme observaçôes efetuadas para a mortalidade por todas as causas;

- a dificuldade em se diagnosticar a diarréia;

- a sua notificação em outro grupo de causas;

- a baixa incidência em alguns municípios, influenciando sobretudo aqueles com reduzida população.

Essa constatação levou a considerar a variável como dicotômica (municípios com ou sem óbitos por doenças diarréicas). Um inconveniente que pode ser levantado para as variáveis dicotômicas, tal como aqui adotadas, é a inclusão na mesma categoria de municípios com taxas de morbi-mortalidade muito pequenas e outros com taxas elevadas, resultando em uma relativa perda de sensibilidade da variável.

O valor médio da mortalidade por doenças diarréicas, calculado por estado conforme apresentado na Tabela 8 , foi certamente influenciado pelos valores nulos de 350 dos 500 municípios considerados. Mesmo assim, a magnitude da mortalidade por doenças diarréicas segue 
Tabela 7 - Mortalidade até cinco anos ( 1999) e mortalidade infantil ( 1998) para os estados de interesse, para o Brasil e para as regiões - valores médios (por mil nascidos vivos)

\begin{tabular}{|c|c|c|c|c|c|c|c|c|c|}
\hline & \multicolumn{2}{|c|}{ Bahia } & \multicolumn{2}{|c|}{ Pernambuco } & \multicolumn{2}{|c|}{ Paraná } & \multicolumn{2}{|c|}{ Rio Grande do Sul } & \multirow{2}{*}{$\begin{array}{c}\text { Brasil } \\
\text { Mortalidade } \\
\text { infantil }\end{array}$} \\
\hline & $\begin{array}{l}\text { Mortalidade } \\
\text { infantil }\end{array}$ & $\begin{array}{c}\text { Mortalidade } \\
\text { até } \\
\text { cinco anos }\end{array}$ & $\begin{array}{l}\text { Mortalidade } \\
\text { infantil }\end{array}$ & $\begin{array}{c}\text { Mortalidade } \\
\text { até } \\
\text { cinco anos }\end{array}$ & $\begin{array}{l}\text { Mortalidade } \\
\text { infantil }\end{array}$ & $\begin{array}{c}\text { Mortalidade } \\
\text { até } \\
\text { cinco anos }\end{array}$ & $\begin{array}{l}\text { Mortalidade } \\
\text { infantil }\end{array}$ & $\begin{array}{l}\text { Mortalidade } \\
\text { até } \\
\text { cinco anos }\end{array}$ & \\
\hline $\begin{array}{l}\text { Municípios } \\
\text { considerados }\end{array}$ & 54 & 37 & 77 & 53 & 29 & 26 & 19 & 18 & \\
\hline Estado & 51 & - & 77 & - & 28 & - & 19 & - & 36,10 \\
\hline Região & 58 & - & 58 & - & 22 & - & 25 & - & \\
\hline
\end{tabular}

a mesma ordem, por estado, dos dois indicadores de mortalidade anteriores.

Mesmo considerando o número de municípios sem registro de óbitos, as correlações da mortalidade por doenças diarréicas em crianças menores de cinco anos com a mortalidade infantil e com a mortalidade por todas as causas até cinco anos $(\mathrm{r}=0,51$ e 0,39 ; respectivamente) poderiam ser classificadas como moderadas.

Vale ressaltar também o alto percentual de municípios nos estados do Nordeste que apresentaram óbitos por doenças diarréicas. Em Pernambuco e na Bahia, 46\% e 72\% dos municípios apresentaram óbitos por doenças diarréicas, respectivamente, enquanto que nos estados da região Sul, apenas 10\% dos municípios, aproximadamente, apresentaram óbitos.

A despeito da fragilidade do indicador, motivada pela proporção de municípios que não apresentaram óbitos, mas considerando sua boa correlação com a mortalidade infantil e com a mortalidade por todas as causas, por um lado, e a forte relação apontada na revisão da literatura entre a mortalidade por doenças diarréicas e ações de saneamento, por outro, indicam que esse indicador deva ser considerado como um indicador importante para representar os efeitos da má prestação de serviços de saneamento ou até mesmo da inexistência deles. Em vista disso, o setor saúde deveria atuar fortemente para melhorar a qualidade dessas informaçôes.

$\mathrm{O}$ indicador sanitário que apresentou associação estatística significativa e em sentido inverso à mortalidade por doenças diarréicas até cinco anos foi o cloro residual (variável dicotômica que diferencia os municípios que têm $100 \%$ das amostras com cloro residual dentro dos padrôes na rede de distribuição de água daqueles que têm percentual diferente de $100 \%)$. Contudo, cobertura por coleta de esgotos e cobertura por coleta de lixo resultaram estatisticamente associadas,

Tabela 8 - Média da mortalidade por doenças diarréicas até cinco anos ( 1999) e média da mortalidade por todas as causas até cinco anos (1999) para os estados de interesse

\begin{tabular}{lc} 
Indicador & \multicolumn{2}{c}{ Estado } \\
Bahia Pernambuco Paraná Rio Grande do Sul
\end{tabular}

\begin{tabular}{lcccc}
\hline $\begin{array}{l}\text { Mortalidade por todas as causas até } \\
\text { cinco anos (por mil) }\end{array}$ & 37 & 53 & 26 & 18 \\
$\begin{array}{l}\text { Mortalidade por doenças diarréicas } \\
\text { até cinco anos (por mil) }\end{array}$ & 1,77 & 4,7 & 0,5 & 0,08 \\
$\begin{array}{l}\% \text { de municípios com óbitos por } \\
\text { diarréia }\end{array}$ & 46 & 72 & 12 & 11 \\
\hline
\end{tabular}

porém com relação direta, tendo sido encontrada uma fraca associação para a coleta de lixo, diminuindo a importância do resultado.

\section{- Morbidade por doenças} diarréicas até cinco anos, decorrentes das internações hospitalares

Os problemas de sub-notificação identificados para os indicadores de mortalidade repetem-se para os indicadores de morbidade, particularmente aqueles referentes à dificuldade de diagnóstico, já destacados para a mortalidade por doenças diarréicas.

Ainda que em número inferior ao da mortalidade por doenças diarréicas, a morbidade por doenças diarréicas apresentou uma proporção significativa de municípios sem internação hospitalar por essa causa: $141(28,2 \%)$ dos 500 municípios considerados. Esse fator levou a tratar também esta variável como dicotômica (municípios com ou sem internações por doenças diarréicas).

$\mathrm{O}$ valor médio da morbidade no estado do Rio Grande do Sul apresentou-se superior ao dos estados de Pernambuco e Paraná. Considerando que a mortalidade do estado do Rio Grande do Sul, nos três indicadores adotados, foi a menor de todas, e supondo-se que este quadro parece não refletir a realidade, deve-se ressaltar aqui os problemas apontados nos itens anteriores relativos aos sub-registros. Tal fato torna vulnerável o indicador de morbidade por internações hospitalares. Alguns estados podem ter melhor qualidade no registro das internaçóes hospitalares, o que resulta em valores mais elevados para a morbidade; entretanto, isto não significa que nos outros estados o número de internações seja, na realidade, inferior.

Outro aspecto que chama a atenção é a baixa correlação entre a morbidade por doenças diarréicas, a partir das internações hospitalares, e as mortalidades até cinco anos por todas as causas, até cinco anos por doenças diarréicas e infantil. Em todos os casos a correlação entre esses indicadores foi considerada como muito fraca (ver Tabela 3). Nem sempre, aliás, na maioria dos casos, as internaçôes acabam evoluindo a óbito, mas mesmo assim seria de se esperar uma melhor associação entre a morbidade e a mortalidade por causa. Essa baixa correlação é mais uma evidência da vulnerabilidade deste indicador epidemiológico. Por outro lado, é sabido que a qualidade do atendimento hospitalar influencia a taxa de óbitos por determinada causa.

A perda de sensibilidade da variável, pela sua natureza dicotômica, 
analogamente à mortalidade por doenças diarréicas, deve ser também registrada.

$\mathrm{O}$ único indicador sanitário que apresentou associação estatística significativa e em sentido inverso à mortalidade por doenças diarréicas até cinco anos foi o cloro residual.

\section{- Morbidade por doenças} diarréicas decorrentes das notificações em unidades de saúde - MDDA

Os dados utilizados para compor este indicador foram fornecidos pelas Secretarias de Saúde dos Estados, por intermédio da FUNASA/MS.

A Monitorização das Doenças Diarréicas Agudas - MDDA é um programa do então Centro Nacional de Epidemiologia-CENEPI/MS, hoje Secretaria de Vigilância em Saúde/MS, em que as unidades de saúde dos municípios monitoram as diarréias, por semana epidemiológica, permitindo assim o acompanhamento de eventuais surtos de diarréia, por qualquer motivo.

Apesar de se trabalhar insistentemente na obtenção de dados dos diversos estados que compunham o Piloto para implantação do SISAGUA, o estado de Pernambuco foi o estado que maior quantidade de dados forneceu (dados de 85 municípios), enquanto o estado da Bahia apresentou dados de 22 municípios e o do Rio Grande do Sul de apenas cinco municípios. O estado do Paraná, apesar de ter a monitorização em vários municípios do estado, não conseguiu preparar os dados por faixa etária a tempo do encerramento do presente trabalho, o que comprometeu a utilização dos seus dados.

Um aspecto importante da análise desse indicador é que sua correlação com as mortalidades infantil e até cinco anos por todas as causas mostrou-se muito bai$\mathrm{xa}$, ao passo que a correlação com a mortalidade até cinco anos por doenças diarréicas, mesmo sendo baixa $(r=0,228)$, é bem mais elevada que a correlação entre a morbidade por doenças diarréicas decorrentes das internaçōes hospitalares e a mortalidade por doenças diarréicas $(r=0,09)$. Isto sugere, mais uma vez, que esse indicador parece ser mais consistente que a morbidade relativa às internações hospitalares.

Os aspectos analisados nos parágrafos anteriores, adicionados ao fato de que os dados da monitorização por doenças diarréicas são obtidos de fontes primárias, sugerem que o indicador morbidade por doenças diarréicas calculado a partir de dados da MDDA seja considerado como potencial indicador epidemiológico, representativo das morbidades nas análises com indicadores sanitários e sociais.

O único indicador sanitário que apresentou associação estatística significativa e em sentido inverso à morbidade por doenças diarréicas decorrentes do monitoramento de doenças diarréicas agudas foi o percentual da população do município com sistemas de abastecimento de água com desinfecção, sendo que o coeficiente de correlação encontrado foi superior $(0,472$ versus 0,332$)$ ao do indicador anterior (morbidade por diarréia em menores de cinco anos decorrente de internações hospitalares), ressaltando a melhor associação.

\section{CONCLUSÃO E CONSIDERAÇỐES FINAIS}

O indicador epidemiológico de base municipal, relativo à mortalidade, que demonstrou maior confiabilidade e consistência foi a mortalidade infantil, pois a metodologia de cálculo foi desenvolvida exatamente para absorver as vulnerabilidades do sub-registro de nascimento e óbito das fontes oficiais.

Entretanto, em função do fato de que esse indicador não considera os óbitos de crianças maiores de um e menores de cinco anos, a mortalidade por todas as causas em crianças menores de cinco anos, deve ser considerada como alternativa, com a ressalva de que se verificam subnotificaçôes significativas e diferenciais entre regiōes do país.

Ainda com a finalidade de se selecionar um indicador de mortalidade que representasse as causas específicas do grupo das doenças diarréicas, pode-se sugerir que a mortalidade por doenças diarréicas em crianças menores de cinco anos é um importante indicador epidemiológico. As ressalvas relativas à sub-notificação cabem também para esse indicador, cujo comportamento é típico de variável dicotômica, do tipo: municípios com óbitos ou sem óbitos.

Quanto ao grupo da morbidade por doenças diarréicas, destaca-se o indicador morbidade por doenças diarréicas em crianças menores de cinco anos decorrente da monitorização de doenças diarréicas agudas - MDDA de unidades de saúde dos municípios. Os motivos da importância desse indicador são detalhados no corpo do presente texto e, resumidamente, seu melhor desempenho em relação à morbidade por doenças diarréicas decorrente das internaçôes hospitalares é possivelmente função de inconsistências deste último e da melhor qualidade das correlações do primeiro com os indicadores sanitários.

Espera-se que as instituições responsáveis pela gestão das informações do setor saúde progressivamente superem as questôes que comprometem a sub-notificação dos registros de nascimento e de óbitos, sobretudo nas regiōes do Norte e Nordeste do país. Resolvidos esses problemas poderá se mostrar mais conveniente a adoção dos indicadores de mortalidade.

Outra recomendação decorrente deste trabalho é a de que a vigilância epidemiológica das três esferas de governo continue apoiando e agilizando o sistema de informação do programa de monitorização das doenças diarréicas agudas (MDDA), pois em princípio parece que o programa, ao trabalhar com dados primários em unidades infra-municipais (unidades de saúde), fornece importantes indicadores e permite agir preventivamente contra tais doenças por intermédio da integração com o setor saneamento com açôes locais básicas. Os resultados da regressão multivariada, ainda que em apenas 112 municípios com dados disponibilizados, dos 500 da amostragem global, mostraram coerência na qualidade da correlação da morbidade por essa fonte com os indicadores sanitários e sócio-econômicos.

\section{AGRADECIMENTOS}

Os autores agradecem à OPAS, por intermédio da Representação no Brasil, em nome da Engenheira Jacira Cancio e à Fundação Nacional de Saúde, por meio da então Coordenação Geral de Vigilância Ambiental em Saúde - CGVAM/ CENEPI//FUNASA/MS, pelo suporte, apoio técnico-científico e incentivo à elaboração do presente estudo, que se baseia na dissertação de mestrado do primeiro autor, defendida junto ao Programa de Pós-Graduação em Tecnologia Ambiental e Recursos Hídricos da UNB, em 2002.

\section{REFERÊNCIAS}

BRISCOE, J.; FEACHEM, R.G.; RAHAMAN, M.M. Evaluating health impact: water supply, sanitation, and hygiene education. Ottawa: International Development Research Centre, 80p. 1986.

CÂMARA, V. M. et al. Curso de epidemiologia para vigilância ambiental em saúde. Brasília: FUNASA, 224p. 2000. 
COSTA, S. S. Indicadores sanitários como sentinelas na promoção da saúde, prevenção e controle de doenças e agravos relacionados ao saneamento: uma experiência a partir do Sistema de Informação de Vigilância e Controle da Qualidade da Água para Consumo Humano no Brasil - o SISAGUA. Dissertação de Mestrado. Publicação PTARH.DM - 52/2002, Departamento de Engenharia Civil e Ambiental, Universidade de Brasília, 169 p. 2002.

ESREY, S. A. et al. Health benefits from improvements in water supply and sanitation: survey and analysis of the literature on selected diseases. WASH (Water and Sanitation for Health Project) Technical Report 66. Washington: U.S. Agency for International Development, 74p. 1990.

ESREY, S. A.; FEACHEM, R. G.; HUGHES J.M. Interventions for the control of diarrhoeal diseases among young children: improving water supplies and excreta disposal facilities. Bulletin of the World Health Organization, 63(4), 757 772. 1985

FUNASA. Sistema de informação sobre mortalidade - 1999. (Informação em meio magnético). 2001a.
FUNASA. Sistema de informação sobre nascidos vivos - 1999. (Informação em meio magnético). $2001 \mathrm{~b}$

HELLER, L. Saneamento e saúde. Brasília: OPAS - Organização Pan-americana da Saúde, 97p. 1997.

MINISTÉRIO DA SAÚDE. Sistema de informação sobre morbidade hospitalar do SUS - CID 10. ht t p: // www.dat a sus.gov.br/cgi/ tabcgi.exe?sih/rnba.def. Consultado em julho/ 2001

PRÜSS, A. et al. Estimating the burden of disease from water, sanitation, and hygiene at a global level. Environmental Health Perspectives, v.110, n.5, p.537-542, May 2002.

SIMŌES, C. C. Brasil: estimativa da mortalidade infantil por microrregioes e municipios. Brasília: Ministério da Saúde, Secretaria Executiva, Secretaria de Políticas Públicas, 79p. 1999.
Endereço para correspondência:

Léo Heller

Universidade Federal de Minas Gerais

Dep. de Engenharia Sanitária e

Ambiental

Av. do Contorno, 842 - $7^{\circ}$ Andar 30 I 30-000 Belo Horizonte - MG Brasil

Tel: (3I) 3238-1978

E-mail: heller@desa.ufmg.br 